\title{
Timing and minimal access surgery for sciatica: a summary of two randomized trials
}

\author{
Mark P. Arts • Wilco C. Peul • \\ Leiden - The Hague Spine Intervention Prognostic \\ Study Group (SIPS)
}

Received: 11 February 2011 / Accepted: 17 February 2011 / Published online: 3 March 2011

(C) The Author(s) 2011. This article is published with open access at Springerlink.com

\section{Background}

Sciatica, better defined as sciatic neuralgia or lumbosacral radicular syndrome (LSRS), is a frequently diagnosed debilitating spine disorder with an estimated yearly incidence of 5-10 per 1,000 persons [22]. The societal impact of low back-related disorders is quite high, as they remain the number one cause of work disability in most Western countries. Sciatica manifests itself as radiating dermatome pain regularly accompanied by diminished jerk reflexes, sensory and motor deficits. The most common cause is a herniated lumbar disc, sometimes combined with bony involvement, compressing an exiting nerve root. Less often the radicular pain is caused by a diabetic neuritis, poly-radiculoneuropathy, or tumor. Although lumbar disc surgery is frequently performed, the timing of this intervention and the preferred technique were until recently important points for debate. The performed numbers of low-back surgeries vary widely between and even within countries and the used intervention techniques do seem to be based on personal or societal preferences instead of evidence-based medicine [10]. Currently, scientific study results have been added to medical knowledge making rational approaches for optimal spine care possible.

M. P. Arts $(\bowtie) \cdot$ W. C. Peul

Department of Neurosurgery, Medical Center Haaglanden, PO Box 432, 2501 CK The Hague, The Netherlands e-mail: m.arts@mchaaglanden.nl

W. C. Peul

Department of Neurosurgery, Leiden University Medical Center,

The Hague, The Netherlands

\section{Diagnosis}

Until recently, classical neurological signs of LSRS were used as a so-called accurate diagnostic tool and decision aid to refer patients for radiological confirmation of a pre-test high probability of nerve root compression by a herniated disc fragment. Subsequently, a surgical indication was routinely made by using the same neurological signs and symptoms as well as the correlating radiological morphology as used for diagnosis. Although it is very plausible to expect that a good fitting history of dermatomal radiating leg pain with provocation by the straight leg raising test and concomitant neurological signs will result in excellent results by surgery, scientific data are still lacking. Patients with absent provocation by the straight leg raising test might risk negative advice for subsequent neurosurgical counseling, while, vice versa, positive provocation tests sometimes lead to unrealistically high expectations of surgical outcome. Late in the 20th century, the bed rest trial showed evidence that the diagnostic value of classical neurological signs is not as accurate as was assumed beforehand [18]. The most important diagnostic variable was the dermatomal area of radiating pain. Moreover, in this landmark study, even in patients with a clear-cut radicular syndrome confirmed by a neurologist, a herniated disc could not be confirmed by experienced neuroradiologists in one-third of the cases. This well-performed study raised serious doubts with regard to the diagnostic value of our neurologically trained methods of examination and clinical textbooks. Surgical studies confirmed the lack of consensus with regard to the exact description and diagnosis of the LSRS caused by a herniated disc. Besides the diagnostic inaccuracy, classical signs fail to discriminate between patients that will be cured by nature compared to those that might benefit by surgery. The presumed 
predictive value for a favorable surgical outcome of classically trained neurological signs, such as the famous Lasègue's test, do seem to be worthless. Moreover, no distinction can be made between patients who will have a favorable natural course and those for whom a relative early timed neurosurgical consultation might be more appropriate.

Even though we cannot predict the presence or absence of mechanical nerve root compression, medical doctors still need to rely on the classical neurological distribution of pain and deficit, since empirical evidence suggests that in less-clear-cut sciatic neuralgias, surgical intervention will yield poor prognosis of outcome in the majority of patients. Randomized controlled trials only included patients who, according to neurologists, had classical syndromes and therefore excluded patients with less-clear pain problems or discordance between dermatomal pain and neurological signs. The exclusion of these patients is completely understandable, but it results in severe selection and referral bias, giving rise to differences between study and clinical patient populations. In other words, the results of the randomized studies so far are the best we can achieve at present, but it would be a mistake to generalize these to usual neurosurgical care. Highly sophisticated mathematical and epidemiological methods will hopefully give more insight in the near future. Therefore, the diagnostic description of LSRS is currently still based on classical nomenclature until better prediction rules exist. Most red flags are easily differentiated from uncomplicated herniated discs with lumbar nerve root compression. From a clinical standpoint, however, it is far more difficult to differentiate between a lumbosacral radicular syndrome and atypical leg pain, also called pseudoradicular, motion-segment, or facet joint pain. The latter pain syndromes do have in comparison to LSRS a less well demarcated pain syndrome, but the differences between nerve root pain and facet joint pain might be less clear when patients did develop a chronic pain syndrome. It seems to be common sense that patients with a radiological confirmed herniated disc should not be offered surgery in absence of nerve root symptoms. Reality, however, proves the contrary, as seldom patients in Western society are surgically treated for their herniated disc or spondylosis without a clear LSRS.

\section{Timing of surgery}

The natural course of sciatica due to a lumbar disc herniation is usually favorable and radicular pain will decrease or disappear within 6-12 weeks after onset in $60-80 \%$ of the patients $[18,47]$. For this reason, most clinicians in Western-world countries prescribe conservative treatment to the patients for at least 6 weeks up to 6 months, before offering surgery. Comparative studies of early surgery versus prolonged conservative strategy in patients with acute sciatica had never been performed. A classic randomized controlled trial on surgery versus conservative care by Weber, was executed more than 20 years ago [49]. Although patients treated with surgery had better outcomes at 1 year, results at 4 years and 10 years were similar to patients treated with conservative care. The study, however, had several important methodological flaws and the main shortcoming was the exclusion of patients with intolerable pain. Therefore, studies were needed to elucidate the debate on surgical timing.

The beginning of the 21 st century is focused on intervention-prognostic studies to evaluate the costeffectiveness of spinal interventions compared to prolonged conservative care with special emphasis on the surgical treatment of sciatica. The Sciatica trial, a Dutch multicenter randomized controlled trial designed to accurately estimate the effectiveness of prolonged conservative treatment strategy compared to surgery in patients with 6-12 weeks of persisting disabling sciatica, presented the early pain relief and recovery of function that surgeons and patients observe in regular life [38]. Patients treated with early surgery had similar outcomes at 1 year as those treated with conservative care, although the early surgery group achieved more rapid recovery and pain relief [37]. A carefully prepared subgroup analysis with predefined baseline variables of the aforementioned trial showed that early surgery compared to prolonged conservative care yielded a significant faster rate of recovery in patients with leg pain provoked by sitting [35]. By a Cox proportional hazard model analysis, this was the only variable that significantly interacted with both timings of surgery strategies. In other words, the patient who asks his physician to be excused for not sitting during the visit because of provocation of excruciating nerve root pain, might be better off with an early surgery strategy. To conclude, a simple anamnestic question gives more insight in the appropriate treatment algorithm than a thorough physical examination including nerve root provocative tests like the straight leg-raising maneuver.

At present, we can conclude that the optimal timing of surgery cannot be generalized to every patient, but that it is an individualized decision. Since early surgery quickly relieves sciatica, it is warranted in patients with severe disabling leg pain. On the other hand, prolonged conservative care is defendable in patients with leg pain not interfering their daily activities. At present, the surgical decision process is more likely to be based on preferences of surgeons rather than the factor duration of sciatica. Sensitivity analysis of the cost-effectiveness study presented early surgery to be an extremely expensive method from a health care economic perspective [46]. The same analysis, however, showed a favorable result of an early 
surgery strategy from a societal perspective. Macroeconomic horizons in Western societies even benefit from an early surgery strategy in the Western world. Instead of relying on preferences of local neurologists, rheumatologists, orthopedic, or spinal neurosurgeons and insurance companies, it seems more appropriate to let the well-informed patient decide about the timing of their intervention.

\section{Surgical techniques}

After the first publication in 1934, lumbar disc surgery became one of the most frequently performed surgical procedures worldwide [29]. The technique described by Mixter and Barr involved extensive removal of the bony lamina with transdural excision of the herniated disc. The rational for opening the dura was twofold; the removal of the irritating fluid lipiodol, which was injected intrathecally for imaging purposes, and the hypothesis that paramedian disc protrusions could not be removed without opening the dura. Love [24] first introduced extradural removal of the lumbar herniated disc by retracting the dura medially and incision of the disc protrusion. For those times, this was a very innovative method, but did not result in worldwide implementation. It was not until the late 1960s that lessinvasive approaches were introduced aiming at shorter hospitalization, less morbidity, and faster recovery. With the introduction of enlightened magnification by loupes or microscope, Yasargil and Caspar launched the unilateral transflaval microdiscectomy, which is presently regarded as the gold standard [7, 52]. Williams popularized microdiscectomy in the United States in 1978. He operated on Las Vegas showgirls and his series of patients documented minimal scars and faster return to work [50].

A paradigm shift began with minimally invasive approaches to the spine. The rational behind minimally invasive spine surgery is less tissue damage, shorter hospitalization, and faster recovery while achieving a good clinical outcome comparable or even better than conventional surgery. Minimally invasive spine surgery has adopted several techniques from other fields and has been influenced by endoscopy, biochemical advances, lasers, and image-guidance systems. In 1963, Smith et al. were the first to inject chymopapain into a herniated disc for the purpose of hydrolyzing the mucoprotein [42, 43]. Although it has been used for more than 30 years, meta-analyses concluded that chemonucleolysis is more effective than a placebo, but less effective than surgical nerve root decompression [15]. In 1975, Hijikata reported percutaneous nucleotomy by inserting a 7-mm-diameter tube under local anesthesia with partial resection of disc material [17]. Kambin [20] reported the first results of arthroscopic discectomy by using a cannula and forceps, which was later refined by Maroon and Onik [25] using a guillotine-like probe into the disc. Choi and Ascher reviewed the first results of percutaneous laser disc decompression aiming at decreasing intradiscal pressure and subsequent nerve root relief [11]. In 1992, Mayer introduced the percutaneous endoscopic laser discectomy, combining forceps and laser [26]. The concept of posterolateral endoscopic discectomy changed from central nucleotomy to transforaminal nerve root decompression. Yeung and Hoogland are credited for the development of the Yeung Endoscopic Spine System (YESS) in 1997 and the Thomas Hoogland Endoscopic Spine System (THESSYS) in 1994, respectively [19, 53]. A recently published systematic review showed no significant differences in outcome between transforaminal endoscopic surgery and conventional microdiscectomy [30].

In 1997, Foley and Smith replaced the subperiosteal muscle dissection of the open microdiscectomy by the transmuscular muscle splitting technique of microendoscopic discectomy [13]. Because of the unfamiliarity of most neurosurgeons with endoscopic techniques, Smith and Foley introduced a modified set of tubular dilators and retractors used specifically for the microscope. Nowadays, thousands of patients have been operated by tubular discectomy in public and private hospitals, mainly stimulated by the publicity and marketing tools of the industry. However, the literature regarding minimally invasive spine surgery is criticized as being overly optimistic and scientific proof supporting the superiority of minimally invasive techniques is lacking. Therefore, every new technique should be compared with the gold standard by means of randomized controlled trials prior to implementation the new procedure on a large scale. One of these so-called Health Technology Assessment (HTA) studies is the Dutch Sciatica-MED trial, which is a multicenter double-blind randomized cost-effectiveness study comparing patients treated by the microtubular method with those treated by conventional microdiscectomy [5].

Whenever a new procedure is to be compared to the gold standard, the latter should be defined. In 1977, Yasargil and Caspar introduced the microscope for surgical removal of lumbar herniated disc [7, 52]. Unilateral transflaval microdiscectomy enabled the use of smaller incisions and facilitated a less traumatic procedure compared with the previously performed bilateral muscle dissection and bony decompression [50, 51]. However, randomized controlled trials on standard macrodiscectomy versus microdiscectomy documented equal hospital stay and postoperative morbidity with no significant differences in outcome [16, 21, 23, 45]. Microsurgical techniques provide optimized illumination and facilitate teaching, but lengthen the surgical procedure. There is moderate evidence that both procedures yield broadly comparable outcomes [15]. A prospective randomized trial on patients undergoing micro- 
discectomy by using either the microscope or loupes magnification (with or without headlight) has never been performed. Generally, unilateral transflaval discectomy with loupes or microscopic magnification are both considered as microdiscectomy and internationally defined as the standard method of treatment of lumbar disc-related sciatica. This is supported by a Canadian and Dutch survey held among spine surgeons showing that the majority of the respondents performed unilateral transflaval microdiscectomy as the standard surgical procedure in their practice $[6,8]$.

\section{Is minimally invasive surgery minimally invasive and maximally effective?}

Minimally invasive treatments of lumbar disc herniations have recently been popularized. Based on the hypothesis "small being better", efforts have been made to decrease tissue damage through smaller corridor approaches. The concept of minimally invasive spine surgery comprises reduced muscle injury while achieving a good clinical outcome comparable with conventional open surgery. Patients are expected to have less low-back pain, shorter hospitalization, faster mobilization and recovery, and quicker resumption of work and daily activities. Additionally, as a result of the hypothetically reduced absence from work, cost-effectiveness is expected to be in favor of minimally invasive surgery.

The first part of this concept, i.e., reduced muscle injury, raises some questions and can be disputed. Is it really true that insertion of tubes through the multifidus muscle nicely split the muscle fibers in a longitudinal direction without tissue damage? Does conventional transection of the lumbodorsal fascia from the spinous process with subperiosteal muscle dissection result in substantial muscle injury? If so, is there a relationship between muscle injury and postoperative low-back pain?

In general, muscle injury can be quantified by the enzyme creatine phosphokinase (CPK) in serum and semiquantified by evaluation of muscle atrophy on MRI. A clear dose-response relationship between the CPK ratio (that is the difference within one patient) and the extent of surgical invasiveness after various spinal surgical approaches has been shown [4]. For example, posterior instrumented fusion of two or more vertebral bodies resulted in higher values of CPK ratio as compared to single-level laminectomy. In the Sciatica-MED trial, no statistically significant difference was shown in the CPK ratio in patients treated with tubular discectomy and patients treated with unilateral transflaval microdiscectomy. Additionally, MR images of the lumbar spine 1 year after surgery showed no difference in multifidus muscle atrophy between tubular discectomy and unilateral transflaval microdiscectomy [3]. Therefore, it can be argued whether the transmuscular splitting technique in tubular retractor surgery is less invasive as compared to unilateral subperiosteal muscle dissection with placement of the conventional Caspar spreader. Based on theses results, apparently not. Insertion of different tubes with increasing diameter might even be more invasive since patients treated with tubular discectomy reported higher scores on the visual analogue scale of low-back pain during the follow-up period. However, these differences were small and did not reach the minimal clinically important difference of $20 \mathrm{~mm}$ $[12,32]$.

The second part of the rational behind minimally invasive surgery, i.e., faster recovery, can also be disputed. No significant difference in postoperative moment of mobilization and length of hospital stay between tubular discectomy and conventional microdiscectomy has been shown [2]. Nearly all patients mobilized the same day of surgery or the day after, and the mean hospital stay in both groups was 3.3 days, including 1 day of pre-operative admission. Patients treated with tubular discectomy and conventional microdiscectomy reported to be fully recovered after a median period of 2 weeks, irrespective of the assigned surgical treatment. Based on these results, it can be concluded that the type of surgical approach does not influence the rate of recovery.

From a societal perspective, cost-utility analysis showed non-significant differences in costs and quality adjusted life years (QALYs) in favor of conventional microdiscectomy. Therefore, it is unlikely that tubular discectomy is more cost-effective than conventional microdiscectomy.

The main reason for the comparable results of conventional microdiscectomy and tubular discectomy is probably the fact that the performed conventional surgery can be defined as minimally invasive as well. For blinding purposes, the participating surgeons did their uttermost best to reduce the skin incision in the conventional group to 25-30 $\mathrm{mm}$, which is smaller than they would have performed in patients not participating in the trial. Conversely, the skin incision in the tubular discectomy group has been extended to $25-30 \mathrm{~mm}$, which is longer than the $18 \mathrm{~mm}$ of the largest tube. Recently, a randomized trial on endoscopic transforaminal discectomy versus conventional unilateral microdiscectomy was performed [40]. The 6-mm skin incision in the transforaminal endoscopic surgery was significantly smaller compared to the conventional microsurgery. Patients of both groups reported similar relief of leg pain, although those treated by the conventional surgery documented more low-back pain during follow-up. The essence of all types of lumbar disc surgery is nerve root decompression, which logically results in equivalent outcome of leg pain and functional status. The result with regard to low-back pain relief remains unsolved as intensity of low-back pain VAS scores seem to decline after surgical 
relieve of nerve root compression, which result is in contrast to prior beliefs.

Knowing that the expected influence of minimally invasive procedures on patients' rate of recovery was not proven, it would be interesting to identify certain subgroups that might benefit more from either tubular discectomy or conventional microdiscectomy. In other words, are there certain patient-related characteristics that might help in the surgical decision-making process? Variables that modified the effect of the treatment strategy on the rate of recovery were type of disc herniation and gender. Patients with contained herniated discs recovered more slowly when tubular discectomy was performed compared to those who underwent conventional microdiscectomy. In case of disc sequestration, there was no difference in rate of recovery between both treatment strategies. Possibly, the corridor in the tubular retractor system in patients with contained disc herniation is insufficient for proper nerve root decompression. Removal of a sequester, however, does not require wide exposure of the disc since the sequester can be mobilized and removed under clear vision make use of a nerve hook retractor. The same argument of insufficient surgical exposure could affect patients with concomitant lateral recess stenosis. A subgroup analysis of the Sciatica MED trial showed a trend that lateral recess stenosis patients reported slower rates of recovery when treated by tubular discectomy compared to those operated by conventional surgery. This difference did not reach statistical significance, which could be explained by the small number of patients with lumbar disc herniation and concomitant lateral recess stenosis [1]. Proponents of minimally invasive surgery strongly believe in the feasibility and success of tubular discectomy and literature reporting bad results is claimed by insufficient experience of the surgeons. Previous data on patients with lumbar spinal stenosis documented safe and effective treatment of minimally invasive bilateral nerve root decompression through a unilateral approach [33]. The authors clearly rejected the argument of a limited surgical exposure.

\section{Learning curve of minimally invasive surgery}

A frequently mentioned factor that might be responsible for differences in outcome is the learning curve of new techniques, tubular discectomy in particular. Correct placement of the tubular dilators, recognition of the anatomical landmarks, and the use of instruments through the tubular retractor represent some of the challenges that a starting surgeon must go through. Repeating a new task will result in improved ability to perform that task, although the final performance may vary between individual surgeons. The steady-state, or so-called asymptote, is defined as the least number of surgical procedures required in order to feel comfortable, to know the pitfalls, and to avoid complications. The asymptote of tubular discectomy seems to develop at approximately 30 cases using an endoscope and 15 cases using the microscope [28, 31]. For this reason, inexperienced surgeons were not participating in this trial and all surgeons had performed at least 15 tubular discectomies prior to participation. Most of the participating surgeons had experience in lumbar disc surgery for more than 5 years with at least 50 procedures (either conventional or tubular) per year.

Surgical time of any new technique will decrease over the course of the learning curve, and assessment of its efficacy against the gold standard will be affected by when the comparison occurred on the learning curve [39]. In our trial, the mean operation time of tubular discectomy was 47 min, which is less than the $60 \mathrm{~min}$ mentioned in the assessment of the learning curve by McLouphlin and Fourney [28]. Our operation time was also shorter than the mean 57 min documented by Parikh et al., who reported their operative results after 4 years experience in tubular discectomy [34]. Analysis of our data showed a trend towards a larger difference in effectiveness in favor of conventional microdiscectomy when patients were operated by the most experienced surgeons compared with those patients operated by less-experienced surgeons. Possibly, the most experienced surgeons performed tubular discectomy too routinely and less precisely, compared to those surgeons who just passed the learning curve. This trial, however, was not powered to perform a multilevel analysis between the 14 surgeons of seven participating hospitals.

Some nuances in the learning curve of tubular discectomy should be made. The assumed asymptote of 15 procedures is based on the learning curve of one individual surgeon [28]. Surgical skills may vary between surgeons; one individual may experience a procedure harsh and time consuming while others feel confident quickly and expand their indication. Whenever the steady-state of a new technique would require more than 100 procedures before getting confident, this "novel" technique might be inappropriate, since it should be practical and manageable by most surgeons. An assessment of the learning curve of a population of surgeons has never been performed but would be more reliable.

In principle, conventional surgery is based on accepted standards and experience and is assumed to have reached its steady-state. Surgeons, or residents, are trained the standard approach of different pathologies and new techniques will be learned once they have become familiar with the conventional technique. Therefore, surgeons performing a new technique will always have less experience as compared to performing the gold-standard procedure, no matter how many procedures. 


\section{Repeated surgery and unfavorable outcome}

Although the vast majority of patients treated with firsttime discectomy have favorable outcomes, same-level recurrent disc herniation may complicate the outcome in a subset of patients. The reported rates of recurrent disc herniation vary between 6 and 13\%, depending on the duration of follow-up, surgical technique, and extent of disc removal [48]. A recently published meta-analysis reported higher rates of recurrent disc herniation after limited discectomy (7\%) than after aggressive discectomy (3.5\%) [27]. In our trial, during the first 2 years of followup, the reoperation rate was $15 \%$ after tubular discectomy and $10 \%$ after conventional microdiscectomy, which was not statistically significantly different. The reason for repeated surgery was recurrent disc herniation in the majority of patients. The high recurrence rate after tubular discectomy was in accordance with the results of Teli et al., who randomized patients with herniated discs into tubular discectomy, microdiscectomy using the microscope, and open discectomy using loupe magnification [44]. However, the recurrence rate after conventional microdiscectomy in this trial is surprisingly high, and unexpected. Possibly, the Sciatica-MED trial patients with short-lasting recurrent leg pain underwent an early postoperative MRI, which resulted in a more aggressive strategy with subsequently higher re-operation rates as one would have expected in the usual care. Secondly, recurrent disc herniation could be related to the extent of disc removal. In all cases, as much of the extruded disc was removed as was necessary, but aggressive subtotal discectomy was never intended. Limited exposure during tubular discectomy may result in a higher recurrence rate, since less disc material was removed compared to conventional microdiscectomy, although the difference was not statistically significant.

Based on the results of the presented studies, the outcome of lumbar disc surgery is not always as successful as we would have expected. Especially the above rate of repeated surgery is in line with most surgeons' experiences. We do, however, fail to acknowledge the enormous problems patients do face with regard to their work and private lives after a failed sciatica treatment. With all good purposes of the above studies, we could not prevent that $20 \%$ of the patients were completely disabled after 2 years. It is not surprisingly that these studies reveal the "best" populations of patients and therefore the actual disability rate of low back-related disorders is probably much higher. As neurosurgeons, we might not be impressed by this disability rate, but from a societal perspective this socalled "benign disc" disease results in an unacceptably high work compensation reimbursement.

\section{Future perspective}

Is tubular discectomy the new gold standard or is it a transient fashion? Whenever the outcomes of tubular discectomy and conventional surgery are similar, can the tubes be wrapped up and put back in the closet like oldfashioned clothes? Certainly not. Regardless of the equal outcome documented by this trial, there still remains a place for tubular discectomy in patients with lumbar disc herniation. Morbidly obese patients with lumbar herniated discs may benefit from tubular discectomy since wide surgical exposure of conventional surgery is prevented and the involved disc is approached directly by the tubular retractor. However, our subgroup analyses did not support this hypothetical advantage, which could be explained by the small number of extreme obese patients in the trial. Patients with far lateral extraforaminal disc herniation may also benefit when treated by microscopic tubular discectomy, since wide midlines approaches with muscle retraction is prevented $[9,14]$. Tubular retractors can also be used in percutaneous pedicle screw fixation with transforaminal interbody fusion. Clinical outcome is similar to conventional open-fusion techniques although blood loss and postoperative pain was significantly lower [41].

Tubular discectomy is not superior to conventional microdiscectomy and the small differences in outcome are favorable to conventional surgery, although not clinically relevant. Therefore, surgical decision-making should be based on the preferences of patients and surgeons. Patients with contained discs or lateral recess stenosis could be warned that tubular discectomy might result in slower recovery. In case of disc sequestration, either technique will result in similar recovery. Whenever a surgeon's standard procedure in all patients with lumbar disc hernia means tubular discectomy, he or she may continue doing so although proclaiming superior results of tubular discectomy should be prevented. One of the main advantages of evidence-based medicine is counseling patients honestly so they can decide which treatment is best for them. Subjective media and industry-driven medicine should be discouraged at all times, and new technologies should only be used on a large scale whenever comparative studies with the gold standard have been performed. From an economical perspective, tubular discectomy is not likely to be costeffective compared to conventional microdiscectomy. Therefore, hospitals and private clinics should be warned against higher charges of minimally invasive techniques proclaiming better results.

Patients with lumbar disc herniation undergoing surgery should also be informed about recurrent disc herniation and unfavorable outcome. In our study, $23 \%$ of the patients treated with conventional surgery and $29 \%$ of the patients treated with tubular discectomy reported a bad outcome at 2 
years after surgery, which is in accordance to the literature [36]. More efforts should be made in the prevention of unfavorable outcome instead of the introduction of new surgical gadgets. Spontaneous disc regeneration has been observed and biological repair of the intervertebral disc by injection of activator proteins, biomaterials, or complex cell matrix composites represent some of these new strategies. Finally, implantation of a barrier to prevent recurrent disc herniation or transplantation of intervertebral disc with adjacent endplates may be a promising solution in the near future.

\section{Conclusions}

In short, we have summarized the latest scientific evidence regarding timing and type of surgery in patients with lumbar disc herniation. Long-term functional outcome in patients with herniated lumbar disc treated with surgery is equal to prolonged conservative care, although patients undergoing surgery recover twice as fast. Whenever patients are treated surgically, there is no evidence of minimally invasive tubular discectomy being superior to conventional unilateral transflaval microdiscectomy. The modest differences in outcome were not clinically relevant for the patients and do not warrant the transition from conventional microdiscectomy to less-invasive approaches. Decision-making should be based on patients' and surgeons' preferences rather than the overly optimistic argument of "small being better".

\section{Conflicts of Interest None.}

Open Access This article is distributed under the terms of the Creative Commons Attribution Noncommercial License which permits any noncommercial use, distribution, and reproduction in any medium, provided the original author(s) and source are credited.

\section{References}

1. Arts MP, Brand R, Koes BW, Peul WC (2010) Effect modifiers of outcome of surgery in patients with herniated disc related sciatica? A subgroup analysis of a randomised clinical trial. J Neurol Neurosurg Psychiatry 81:1265-1274

2. Arts MP, Brand R, van den Akker ME, Koes BW, Bartels RH, Peul WC (2009) Tubular discectomy vs. conventional microdiscectomy for sciatica: a randomized controlled trial. JAMA 302:149-158

3. Arts MP, Brand R, van der Kallen B, Lycklama a Nijeholt G, Peul WC (2011) Does minimally invasive lumbar disc surgery results in less muscle injury than conventional surgery? A randomized controlled trial. Eur Spine J 20:51-57
4. Arts MP, Nieborg A, Brand R, Peul WC (2007) Serum creatine phosphokinase as an indicator of muscle injury after various spinal and nonspinal surgical procedures. J Neurosurg Spine 7:282-286

5. Arts MP, Peul WC, Brand R, Koes BW, Thomeer RT (2006) Costeffectiveness of microendoscopic discectomy versus conventional open discectomy in the treatment of lumbar disc herniation: a prospective randomised controlled trial [ISRCTN 51857546]. BMC Musculoskelet Disord 7:42

6. Arts MP, Peul WC, Koes BW, Thomeer RT (2008) Management of sciatica due to lumbar disc herniation in the Netherlands: a survey among spine surgeons. J Neurosurg Spine 9:32-39

7. Caspar W (1977) A new surgical procedure for lumbar disk herniation causing less tissue damage through a microsurgical approach. Adv Neurosurg 4:74-77

8. Cenic A, Kachur E (2009) Lumbar discectomy: a national survey of neurosurgeons and literature review. Can J Neurol Sci 36:196-200

9. Cervellini P, De Luca GP, Mazzetto M, Colombo F (2005) Microendoscopic-discectomy (MED) for far lateral disc herniation in the lumbar spine. Technical note. Acta Neurochir Suppl 92:99-101

10. Cherkin DC, Deyo RA, Loeser JD, Bush T, Waddell G (1994) An international comparison of back surgery rates. Spine 19:1201-1206

11. Choy DS, Ascher PW, Ranu HS, Saddekni S, Alkaitis D, Liebler W, Hughes J, Diwan S, Altman P (1992) Percutaneous laser disc decompression. A new therapeutic modality. Spine 17:949-956

12. Farrar JT, Portenoy RK, Berlin JA, Kinman JL, Strom BL (2000) Defining the clinically important difference in pain outcome measures. Pain 88:287-294

13. Foley KT, Smith MM (1997) Microendoscopic discectomy. Techn Neurosurg 3:301-307

14. Foley KT, Smith MM, Rampersaud YR (1999) Microendoscopic approach to far-lateral lumbar disc herniation. Neurosurg Focus 7:5E5

15. Gibson J, Waddell G (2007) Surgical interventions for lumbar disc prolapse. Cochrane Database Syst Rev:CD001350

16. Henriksen L, Schmidt K, Eskesen V, Jantzen E (1996) A controlled study of microsurgical versus standard lumbar discectomy. Br J Neurosurg 10:289-293

17. Hijikata S (1989) Percutaneous nucleotomy. A new concept technique and 12 years' experience. Clin Orthop:9-23

18. Hofstee DJ, Gijtenbeek JM, Hoogland PH, van Houwelingen HC, Kloet A, Lotters F, Tans JT (2002) Westeinde Sciatica Trial: randomized controlled study of bed rest and physiotherapy for acute sciatica. J Neurosurg 96:45-49

19. Hoogland T, Schubert M, Miklitz B, Ramirez A (2006) Transforaminal posterolateral endoscopic discectomy with or without the combination of a low-dose chymopapain: a prospective randomized study in 280 consecutive cases. Spine 31:890-897

20. Kambin P, Schaffer JL (1989) Percutaneous lumbar discectomy. Review of 100 patients and current practice. Clin Orthop 238:24-34

21. Katayama Y, Matsuyama Y, Yoshihara H, Sakai Y, Nakamura H, Nakashima S, Ito Z, Ishiguro N (2006) Comparison of surgical outcomes between macro discectomy and micro discectomy for lumbar disc herniation: a prospective randomized study with surgery performed by the same spine surgeon. J Spinal Disord Tech 19:344-347

22. Konstantinou K, Dunn KM (2008) Sciatica: review of epidemiological studies and prevalence estimates. Spine 33:2464-2472

23. Lagarrigue J, Chaynes P (1994) Comparative study of disk surgery with or without microscopy. A prospective study of 80 cases. Neurochirurgie 40:116-120

24. Love JG, Camp JD (1937) Root pain resulting from intraspinal protrusion of intervertebral discs: diagnosis and surgical treatment. J Bone Joint Surg Am 19:776-804

25. Maroon JC, Onik G (1987) Percutaneous automated discectomy: a new method for lumbar disc removal. Technical note. J Neurosurg 66:143-146 
26. Mayer HM, Brock M, Berlien HP, Weber B (1992) Percutaneous endoscopic laser discectomy (PELD). A new surgical technique for non-sequestrated lumbar discs. Acta Neurochir Suppl Wien 54:53-58

27. McGirt MJ, Ambrossi GL, Datoo G, Sciubba DM, Witham TF, Wolinsky JP, Gokaslan ZL, Bydon A (2009) Recurrent disc herniation and long-term back pain after primary lumbar discectomy: review of outcomes reported for limited versus aggressive disc removal. Neurosurgery 64:338-344

28. McLoughlin GS, Gregory S, Fourney DR, Daryl R (2008) The learning curve of minimally-invasive lumbar microdiscectomy. Can J Neurol Sci 35(1):75-78

29. Mixter WJ, Barr JS (1934) Rupture of the intervertebral disc with involvement of the spinal canal. N Engl J Med 211:210-215

30. Nellensteijn J, Ostelo R, Bartels R, Peul W, van Royen B, van Tulder M (2010) Transforaminal endoscopic surgery for symptomatic lumbar disc herniations: a systematic review of the literature. Eur Spine J 19:181-204

31. Nowitzke AM (2005) Assessment of the learning curve for lumbar microendoscopic discectomy. Neurosurgery 56:755-762

32. Ostelo RW, de Vet HC (2005) Clinically important outcomes in low back pain. Best Pract Res Clin Rheumatol 19:593-607

33. Palmer S, Turner R, Palmer R (2002) Bilateral decompression of lumbar spinal stenosis involving a unilateral approach with microscope and tubular retractor system. J Neurosurg 97:213217

34. Parikh K, Tomasino A, Knopman J, Boockvar J, Hartl R (2008) Operative results and learning curve: microscope-assisted tubular microsurgery for 1- and 2-level discectomies and laminectomies. Neurosurg Focus 25:E14

35. Peul WC, Arts MP, Brand R, Koes BW (2009) Timing of surgery for sciatica: subgroup analysis alongside a randomized trial. Eur Spine J 18:538-545

36. Peul WC, van den Hout WB, Brand R, Thomeer RT, Koes BW (2008) Prolonged conservative care versus early surgery in patients with sciatica caused by lumbar disc herniation: two-year results of a randomised controlled trial. BMJ 336:1355-1358

37. Peul WC, van Houwelingen HC, van den Hout WB, Brand R, Eekhof JA, Tans JT, Thomeer RT, Koes BW (2007) Surgery versus prolonged conservative treatment for sciatica. N Engl J Med 356:2245-2256

38. Peul WC, van Houwelingen HC, van der Hout WB, Brand R, Eekhof JA, Tans JT, Thomeer RT, Koes BW (2005) Prolonged conservative treatment or 'early' surgery in sciatica caused by a lumbar disc herniation: rationale and design of a randomized trial [ISRCT 26872154. BMC Musculoskelet Disord 6:8
39. Ramsay CR, Grant AM, Wallace SA, Garthwaite PH, Monk AF, Russell IT (2001) Statistical assessment of the learning curves of health technologies. Health Technol Assess 5:1-79

40. Ruetten S, Komp M, Merk H, Godolias G (2008) Full-endoscopic interlaminar and transforaminal lumbar discectomy versus conventional microsurgical technique: a prospective, randomized, controlled study. Spine 33(9):931-939

41. Scheufler KM, Dohmen H, Vougioukas VI (2007) Percutaneous transforaminal lumbar interbody fusion for the treatment of degenerative lumbar instability. Neurosurgery 60:203-212

42. Smith L (1964) Enzyme dissolution of the nucleus pulposus in humans. JAMA 187:137-140

43. Smith L, Garvin PJ, Gesler RM, Jennings RB (1963) Enzyme dissolution of the nucleus pulposus. Nature 198:1311-1312

44. Teli M, Lovi A, Brayda-Bruno M, Zagra A, Corriero A, Giudici F, Minoia L (2010) Higher risk of dural tears and recurrent herniation with lumbar micro-endoscopic discectomy. Eur Spine J 19:443-450

45. Tullberg T, Isacson J, Weidenhielm L (1993) Does microscopic removal of lumbar disc herniation lead to better results than the standard procedure? Results of a one-year randomized study. Spine 18:24-27

46. van den Hout WB, Peul WC, Koes BW, Brand R, Kievit J, Thomeer RT (2008) Prolonged conservative care versus early surgery in patients with sciatica from lumbar disc herniation: costutility analysis alongside a randomised controlled trial. BMJ 336:1351-1354

47. Vroomen PC, de Krom MC, Slofstra PD, Knottnerus JA (2000) Conservative treatment of sciatica: a systematic review. J Spinal Disord 13:463-469

48. Watters WC III, McGirt MJ (2009) An evidence-based review of the literature on the consequences of conservative versus aggressive discectomy for the treatment of primary disc herniation with radiculopathy. Spine J 9:240-257

49. Weber H (1983) Lumbar disc herniation. A controlled, prospective study with ten years of observation. Spine 8:131-140

50. Williams RW (1978) Microlumbar discectomy: a conservative surgical approach to the virgin herniated lumbar disc. Spine 3:175-182

51. Wilson DH, Harbaugh R (1981) Microsurgical and standard removal of the protruded lumbar disc: a comparative study. Neurosurgery 8:422-427

52. Yasargil MG (1977) Microsurgical operations for herniated lumbar disc. Adv Neurosurg 4:81

53. Yeung AT, Tsou PM (2002) Posterolateral endoscopic excision for lumbar disc herniation: surgical technique, outcome, and complications in 307 consecutive cases. Spine 27:722-731 